\title{
ADAPTIVE TRACKING CONTROL OF EULER-LAGRANGE NONLINEAR SYSTEMS IN THE PRESENCE OF UNCERTAINTY AND INPUT NOISE WITH GUARANTEED TRACKING ERRORS
}

\author{
NGUYEN VAN CHI ${ }^{1}$, NGUYEN DOAN PHUOC ${ }^{2}$ \\ ${ }^{1}$ Thai Nguyen University of Technology, Vietnam; Email: ngchi@tnut.edu.vn \\ ${ }^{2} \mathrm{Ha}$ Noi University of Science and Technology
}

Tóm tắt. Hệ phi tuyến Euler-Lagrange có đồng thời tham số bất định và nhiễu đầu vào (ENUI) là mô hình của rất nhiều các thiết bị công nghiệp trong thực tế như tay máy robot, hệ cơ khí Tora, hệ cơ điện tử Lavitat v.v. Các công trình nghiên cứu trước đây chủ yếu tập trung cho các bài toán điều khiển ổn định hệ với giả thiết chỉ xét đến tham số bất định hoặc chỉ xét đến nhiễu đầu vào. Trong các tài liệu [1], [2] và [3] chúng tôi đã giới thiệu một phương pháp điều khiển bám quỹ đạo thích nghi mới, phương pháp điều khiển này vừa có khả năng bù được sự ảnh hưởng của tham số bất định và có khả năng giảm thiểu được sự ảnh hưởng của nhiễu đầu vào lên hệ. Mặt khác với phương pháp điều khiển đó, sai lệch bám quỹ đạo sẽ được điều khiển hội tụ về một miền hấp dẫn sai lệch bám nhỏ tùy ý quanh gốc tọa độ. Trong bài báo này chúng tôi tiếp tục cải tiến cách lựa chọn tham số để có thể thay đổi được thời gian quá độ của sai lệch bám quỹ đạo, qua đó chúng ta có thể điều chỉnh được một cách độc lập miền hấp dẫn của sai lệch bám và thời gian quá độ trong bài toán điều khiển ổn định theo sai lệch bám quỹ đạo cho hệ ENUI.

Từ khóa. Hệ phi tuyến, điều khiển thích nghi, điều khiển bám quỹ đạo,ổn định ISS, kháng nhiễu.

\begin{abstract}
The Euler-Lagrange nonlinear system with both uncertain parameters and input noises(ENUI) is a common model of many plants in practice as, robot manipulators, mechanical Tora systems, Lavitat mechanical systems, etc. The previous studies are most oriented to control problems for separate cases: parameters uncertainty or input noises. In papers $[1,2,3]$ we introduced a new adaptive tracking control method based on disturbance attenuation and ISS stabilization of ENUI. This both methods compensate the uncertain parameters and eliminate the effect of noise on the inputs of systems. The advantage of this adaptive tracking control is the ability to converge the tracking error to be arbitrary sufficiently small around the neighbourhood of the origin. In this paper, we continue to present a parameter modifying method of an adaptive tracking controller to get an optional transient time. With this modification, we can adjust independently the dimension of tracking errors attractor and the transient time.
\end{abstract}

Key words. Nonlinear systems, adaptive control, tracking control, ISS stabilization, disturbance attenuation.

\section{INTRODUCTION}

Consider the following dynamic model of ENUI given by 


$$
M(\underline{q}, \underline{\theta}) \underline{\ddot{q}}+\underline{g}(\underline{q}, \underline{\ddot{q}}, \underline{\theta})=\underline{u}+\underline{\eta}(t)
$$

where $\underline{q}=\left(q_{1}, q_{2}, \ldots, q_{n}\right)^{T},\left(\underline{u}=\left(u_{1}, u_{2}, \ldots, u_{n}\right)^{T}\right)$ are output and input vectors, respectively; $\underline{\theta}=\left(\theta_{1}, \theta_{2}, \ldots, \theta_{n}\right)^{T}$ represents time invariant uncertain parameter vector; $\underline{\eta}(t) \in R^{n}$ denotes the input noise vector; $M(\underline{q}, \underline{\theta}) \in R^{n \times n}$ is a positive definite symmetric function matrix depending on $\underline{q}$ and $\underline{\theta}$; vector $\underline{g}(\underline{q}, \underline{\ddot{q}}, \underline{\theta}) \in R^{n}$ is dependent on vector $\underline{q}, \underline{\ddot{q}}$ and $\underline{\theta}$. For system (1), the given matrices have the following properties:

Property 1: $M(\underline{q}, \underline{\theta}) \in R^{n \times n}$ is symmetric and positive definite

Property 2: Model (1) with vector of uncertain constant parameters $\underline{\theta}$ can be written as [4]

$$
M(\underline{q}, \underline{\theta}) \underline{\ddot{q}}+\underline{g}(\underline{q}, \underline{\ddot{q}}, \underline{\theta})=D_{0}(\underline{q}, \underline{\dot{q}}, \underline{\ddot{q}})+D_{1}(\underline{q}, \underline{\dot{q}}, \underline{\ddot{q}}) \underline{\theta} .
$$

In the case if the system is unaffected by input noises $\underline{\eta}(t)=\underline{0}, M(\underline{q}, \underline{\theta}) \underline{\ddot{q}}+\underline{g}(\underline{q}, \underline{\ddot{q}}, \underline{\theta})=\underline{u}$, then the controller introduced in $[4,5,6]$

$$
\underline{u}=\widehat{M}\left(d^{2} \underline{w} / d t^{2}+K_{1} \underline{e}+K_{2} d \underline{e} / d t\right)+\underline{g}
$$

is utilized, where $K_{1}, K_{2}$ are two positive definite symmetric matrices optionally; $\overparen{M}, \widehat{g}$ are the brief notations of $\widehat{M}=\widehat{M}(\underline{q}, \underline{p}), \underline{\widehat{g}}=\widehat{g}(\underline{q}, \underline{\dot{q}}, \underline{p}) ; \underline{w}$ is desired trajectory vector; $\underline{e}=\underline{w}-\underline{q}$ is tracking error vector; $\underline{p}(t)$ is an estimation parameter vector for unknown the parameter vector $\underline{\theta}$; received from adjustment mechanisms

$$
d \underline{p} / d t=Q\left(\widehat{M}^{-1} D_{1}\right)^{T}(\Theta, I) P(\underline{e} d \underline{e} / d t)^{T}(4)
$$

with any positive definite symmetric matrix $Q$ and positive definite root $P$ of the Lyapunov equation

$$
A^{T} P+P A=-Q
$$

where $A=\left(\begin{array}{cc}\Theta & I \\ -K_{1} & -K_{2}\end{array}\right)$ and $\Theta$ is the zero matrix, $I$ is the identity matrix of the same dimension, which are only used for the case of the assumption $\underline{\eta}(t)=\underline{0}$.

In the presence of input noise $\underline{\eta}(t) \neq \underline{0}$, the controller introduced in the paper [5] can drive the tracking errors to a neighbourhood of the origin defined with the quite large radius

$$
r=1+\mu_{1}^{-1}\left[\beta_{1}+2 \beta_{0} \beta_{2}+\beta_{2} c \sqrt{\mu_{1}+\mu_{2}}\right] \geq 1 .
$$

However the controller can be only used under the following assumptions

$$
\mu_{2}^{-1} \leq\left\|M^{-1}\right\| \leq \mu_{1}^{-1} ;\|\Gamma\| \leq 1 ; \Gamma=M^{-1} \widehat{M}^{-1}-I ;\|\underline{\delta}\| \leq \beta_{0}+\beta_{1}\|\underline{e}\|+\beta_{2}\|\underline{e}\|^{2} ; \underline{\delta}=\underline{g}-\underline{g}
$$

where $\mu_{1}, \mu_{2}, \beta_{0}, \beta_{1}, \beta_{2}, c$ are positive constants w.r.t. the estimation $\overparen{M}, \widehat{g}$ for $M, \underline{g}$. 
Using ISS(input stable to state) theory, we introduced a new adaptive tracking control method based on disturbance attenuation and ISS stabilization of ENUI in [1-3]. In this paper, we propose a parameter modification of the earlier developed controller to get a smaller transient time. With this modification, we can adjust independently the dimension of the tracking errors attractor and the transient time of the closed system. The advantage of this method is that the tracking error can converge smoothly to an arbitrary sufficiently small neighbourhood of the origin in optional transient time. In the second section, we introduce an algorithm for adaptive tracking control designing ENUL and present a modification to get an optional transient time. Finally, an example is given to illustrate the proposed algorithm. The paper ends with some conclusions.

\section{ADAPTIVE TRACKING CONTROL BASED ON DISTURBANCE ATTENUATION AND ISS STABILIZATION FOR ENUI}

We replace the uncertain parameter vector $\underline{\theta}$ in $M, \underline{g}$ by an optional estimative constant vector $\underline{p}$. Sufficient small $\underline{\hat{p}}=\underline{\theta}-\underline{p}$ is not required, which means that it does not need to be bounded by the conditions (7) as in [5]. From (3), let us supplement an external signal $\underline{v}(t)$, which will be used later to compensate the noise. From [5] we have the structure of the tracking controller as

$$
\underline{u}=\widehat{M}\left(d^{2} \underline{w} / d t^{2}+K_{1} \underline{e}+K_{2} d \underline{e} / d t\right)+\underline{g}+\underline{v}(t) .
$$

Substitute the controller (8) into (1), we have

$$
M(\underline{q}, \underline{\theta}) \underline{\ddot{q}}+\underline{g}(\underline{q}, \underline{\ddot{q}}, \underline{\theta})=\widehat{M}(\underline{q}, \underline{p})\left(d^{2} \underline{w} / d t^{2}+K_{1} \underline{e}+K_{2} d \underline{e} / d t\right)+\underline{\widehat{g}}(\underline{q}, \underline{\ddot{q}}, \underline{p})+\underline{v}(t)+\underline{\eta}(t) .
$$

Setting $\underline{e}=\underline{w}-\underline{q}, \underline{w}=\underline{e}+\underline{q}$, the equation (9) becomes

$$
\begin{aligned}
& M(\underline{q}, \underline{\theta}) \underline{\ddot{q}}+\underline{g}(\underline{q}, \underline{\ddot{q}}, \underline{\theta})=\widehat{M}(\underline{q}, \underline{p})\left(\frac{d^{2} \underline{e}}{d t^{2}}+\frac{d^{2} \underline{q}}{d t^{2}}+K_{1} \underline{e}+K_{2} \frac{d \underline{e}}{d t}\right)+\underline{\widehat{g}}(\underline{q}, \underline{\ddot{q}}, \underline{p})+\underline{v}(t)+\underline{\eta}(t) \\
&=\widehat{M}(\underline{q}, \underline{p}) \underline{\ddot{q}}+M(\underline{q}, \underline{p})\left(\frac{d^{2} \underline{e}}{d t^{2}}+K_{1} \underline{e}+K_{2} \frac{d \underline{e}}{d t}\right)+\widehat{g}(\underline{q}, \underline{\ddot{q}}, \underline{p})+\underline{v}(t)+\underline{\eta}(t) \\
& \Longleftrightarrow(M(\underline{q}, \underline{\theta})-\widehat{M}(\underline{q}, \underline{p})) \underline{\ddot{q}}+\underline{g}(\underline{q}, \underline{\ddot{q}}, \underline{\theta})-\widehat{g}(\underline{q}, \underline{\ddot{q}}, \underline{p})=\widehat{M}(\underline{q}, \underline{p})\left(\frac{d^{2} \underline{e}}{d t^{2}}+K_{1} \underline{e}+K_{2} \frac{d \underline{e}}{d t}\right)+\underline{v}(t)+\underline{\eta}(t) .
\end{aligned}
$$

or briefly in short

$$
(M-\widehat{M}) \underline{\ddot{q}}+\underline{g}-\widehat{\widehat{g}}=\widehat{M}\left(\frac{d^{2} \underline{e}}{d t^{2}}+K_{1} \underline{e}+K_{2} \frac{d \underline{e}}{d t}\right)+\underline{v}(t)+\underline{\eta}(t) .
$$

With this controller, the closed system describes the following tracking error dynamic 


$$
\begin{aligned}
\left.\frac{d^{2} \underline{e}}{d t^{2}}+K_{1} \underline{e}+K_{2} \frac{d \underline{e}}{d t}\right) & =\widehat{M}^{-1}(M-\widehat{M}) \underline{\ddot{q}}+(\underline{g}-\underline{\widehat{g}})-\underline{v}-\underline{\eta}(t) \\
& =\widehat{M}^{-1}(M \underline{\ddot{q}}+\underline{g}-(\widetilde{M} \underline{\ddot{q}}+\underline{\widehat{g}})-\underline{v}-\underline{\eta}(t))
\end{aligned}
$$

and with the characteristic Property 1 and Property 2 of system (1) we have

$$
\begin{aligned}
\left.\frac{d^{2} \underline{e}}{d t^{2}}+K_{1} \underline{e}+K_{2} \frac{d \underline{e}}{d t}\right) & =\widehat{M}^{-1}\left(D_{0}(\underline{q}, \underline{\dot{q}}, \underline{\ddot{q}})+D_{1}(\underline{q}, \underline{\dot{q}}, \underline{\ddot{q}}) \underline{\theta}\right)-D_{0}(\underline{q}, \underline{\dot{q}}, \underline{\ddot{q}})-D_{1}(\underline{q}, \underline{\dot{q}}, \underline{\ddot{q}}) \underline{p}-\underline{v}-\underline{\eta} \\
& =\widehat{M}^{-1}\left(D_{1}(\underline{q}, \underline{\dot{q}}, \underline{\ddot{q}})(\underline{\theta}-\underline{p})-\underline{v}-\underline{\eta}\right) \\
& =\widehat{M}^{-1}\left(D_{1} \underline{\underline{p}}-\underline{v}-\underline{\eta}\right)
\end{aligned}
$$

where $\widehat{\widehat{p}}=\underline{\theta}-\underline{p}$.

Define $\underline{v}=D_{1} \underline{z}$ and $\underline{x}=(\underline{e} \underline{\dot{e}})^{T}$, then using the form (5) of matrix $A$, the tracking error system becomes

$$
\frac{d \underline{x}}{d t}=A \underline{x}+B\left(D_{1}(\underline{\underline{p}}-\underline{z})-\underline{\eta}\right), B=\left(\Theta \widehat{M}^{-1}\right)^{T}
$$

We use the implicit reference model

$$
d \underline{x}_{m} / d t=A \underline{x}_{m}
$$

where $A$ is stable matrix, because $K_{1}, K_{2}$ are two positive definite symmetric matrices. Then, the specific task for designing the tracking error compensation mechanism is now to determine the disturbance compensation signal $\underline{v}(t)$ so that the error between $\underline{x}(t)$ trajectory $(12)$ and desired trajectory $\underline{x}_{m}(t)$ of $(13)$ converges asymptotically to $\underline{0}$. We take a positive definite function of error between $\underline{x}(t)$ and $\underline{x}_{m}(t)$ as

$$
V=\left(\underline{x}-\underline{x}_{m}\right)^{T} P\left(\underline{x}-\underline{x}_{m}\right)+(\underline{\widehat{p}}-\underline{z})^{T} E(\underline{\underline{p}}-\underline{z})
$$

where $E$ is a positive definite symmetric matrix optionally, $P$ is a positive definite symmetric root of Lyapunov equation (5) and $Q$ is an arbitrary positive definite symmetric matrix. Using (12) and (13) we obtain that $\underline{\underline{p}}=\underline{\theta}-\underline{p}$ is a constant vector. The derivative of (14) along (12) is given by

$$
\begin{aligned}
\frac{d V}{d t} & =\left(\underline{x}-\underline{x}_{m}\right)^{T}\left(A^{T} P+P A\right)\left(\underline{x}-\underline{x}_{m}\right)+ \\
& 2(\underline{\underline{p}}-\underline{z})^{T}\left[D_{1}^{T} B^{T} P\left(\underline{x}-\underline{x}_{m}\right)-E \frac{d \underline{z}}{d t}\right]-2\left(\underline{x}-\underline{x}_{m}\right)^{T} P B \underline{\eta} \\
& =-\left(\underline{x}-\underline{x}_{m}\right)^{T} Q\left(\underline{x}-\underline{x}_{m}\right)+2(\underline{\widehat{p}}-\underline{z})^{T}\left[D_{1}^{T} B^{T} P\left(\underline{x}-\underline{x}_{m}\right)-E \frac{d \underline{z}}{d t}\right]-2\left(\underline{x}-\underline{x}_{m}\right)^{T} P B \underline{\eta}
\end{aligned}
$$


By choosing of $\underline{x}_{m}(t)=\underline{0}, \forall t$, the equation (15) becomes

$$
\frac{d V}{d t}=-\underline{x}^{T} Q \underline{x}+2(\underline{\widehat{p}}-\underline{z})^{T}\left[D_{1}^{T} B^{T} P \underline{x}-E \frac{d \underline{z}}{d t}\right]-2 \underline{x}^{T} P B \underline{\eta} .
$$

When $\eta=\underline{0}$, to keep $d V / d t<0$, and letting $\underline{x} \rightarrow 0, \underline{\dot{x}} \rightarrow 0$, we get adaptive tracking error compensation mechanism (AECM)

$$
\left\{\begin{array}{l}
\frac{d \underline{z}}{d t}=E^{-1} D_{1}^{T} B^{T} P \underline{x} \\
\underline{v}=D_{1} \underline{z}
\end{array}\right.
$$

Next, when $\eta \neq \underline{0}$ we need to improve the quality of the controller (8) and AECM (17) to minimize the affect of external disturbance $\eta \neq \underline{0}$ for the tracking error of $\underline{x}(t)$. Obviously, with more freedom of choice for the matrices $\bar{K}_{1}, K_{2}, Q, E, \underline{p}$ we can do that. We just need to create $d V / d t<0$ when $\underline{x} \notin \Omega$, where $\Omega$ is a small enough neighbourhood around the origin. First, we define

$$
\mu=\|\underline{\eta}\|_{\infty}=\sup _{t}|\underline{\eta}(t)|, \gamma(\underline{q}, \underline{p})=\left\|\widehat{M}^{-1}\right\|_{1}
$$

and choose parameter vector $\underline{p}$ so that $\gamma(\underline{q}, \underline{p})$ reaches the minimum of

$$
\gamma_{\min }=\min _{\underline{p}} \max _{\underline{q}} \gamma(\underline{q}, \underline{p})
$$

Then as in $[5,7]$, we used diagonal matrices $K_{1}=\operatorname{diag}\left(k_{1 i}\right), K_{2}=\operatorname{diag}\left(k_{2 i}\right), k_{2 i}^{2}>$ $k_{1 i}>0, i=1,2, \ldots, n$ and the positive definite matrix $Q=2\left(\begin{array}{cc}2 K_{1} K_{2} & \Theta \\ \Theta & K_{2}^{2}-K_{1}\end{array}\right)$. Using the selected $Q$, the positive definite symmetric root $P$ of equation (5) is given by

$$
P=\left[2 K_{1} K_{2} K_{1} ; K_{1} K_{1}\right]^{T}
$$

and (16) becomes

$$
d V / d t=-2|\underline{x}|\left(\lambda|\underline{x}|-\delta \gamma_{\min } \mu\right)
$$

where

$$
\begin{aligned}
& \delta=\max \left(k_{11}, \ldots, k_{1 n}, k_{21}, \ldots, k_{2 n}\right) \\
& \lambda=\min \left(k_{11}^{2}, \ldots, k_{1 n}^{2}, k_{21}^{2}-k_{11}, \ldots, k_{2 n}^{2}-k_{1 n}\right)
\end{aligned}
$$

The equation (21) shows that if we have $|\underline{x}|>\delta \gamma_{\min } \mu / \lambda$ then $d V / d t$ will be negative definite. In other words, tracking errors always tend to origin, since it is outside the neighbourhood of origin (called the attractor)

$$
\Omega=\left\{\underline{x} \in R^{2 n}|| \underline{x} \mid \leq \delta \gamma_{\min } \mu / \lambda\right\}
$$


The dimension of the above attractor $\Omega$ can be sufficiently adjusted, optionally via the positive diagonal matrices $K_{1}, K_{2}$. Unlike the result we proposed in [1-3], in this paper we choose

$$
\begin{array}{r}
k_{11}=\ldots=k_{1 n}=\varepsilon a, a>0,1 \leq \varepsilon \leq a ; \\
k_{21}=\ldots=k_{2 n}=\sqrt{a(a+1)}
\end{array}
$$

where $\varepsilon, a$ are any bounded real numbers to be chosen. So $\delta=\sqrt{a(a+1)}$ is also a bounded value. Therefore $\lambda=a^{2}$, which means

$$
\lim _{a \rightarrow \infty} \gamma_{\min } \mu \sqrt{a(a+1)} / a^{2}=0
$$

or the attractor dimension converges to zero when $a \rightarrow \infty$. If the more you choose $\varepsilon$ closes to the parameter $a$, the more eigenvalues of matrix $A$ of implicit reference model (13) go far image axis. Therefore, transient time of the closed system is shorter. $1 \leq \varepsilon \leq a$ is a transient time adjusting constant.

The adaptive control system in Fig. 1 with controller (8) and tracking error compensation mechanism (17) will not only compensate the tracking error caused by uncertainty component $\underline{\underline{p}}=\underline{\theta}-\underline{p}$ but also attenuate the effect caused by disturbance $\underline{\eta}(t)$ in the sense of leading the derivation component caused by itself to the sufficient small neighbourhood $\Omega$ of the origin. Transient time of the closed system can be adjusted by choosing constant value of $\varepsilon$.

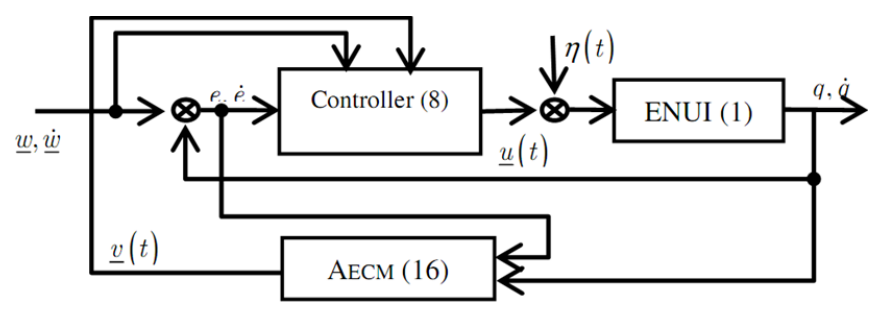

Fig. 1. Adaptive tracking control based on disturbance attenuation and ISS stabilization for systems (1)

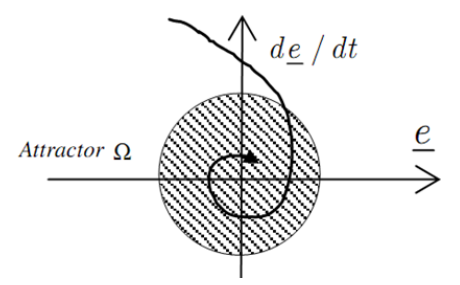

Fig. 2. Attractor of tracking errors

Theorem 1. Assume Properties 1 and 2. The control system in Figure 1 with controller (8) and tracking error compensation mechanism (17), where $a>0$ and $1 \leq \varepsilon \leq a$ are sufficiently adjusted, ensures tracking errors $(\underline{e} d \underline{e} / d t)^{T}$ always tend to the neighbour of the origin. The dimension of the attractor (23) converges to zero when $a=\infty$. Transient time of closed system can be adjusted by choosing constant value of $\varepsilon$. 


\section{APPLYING EXAMPLE}

In order to show the results of the proposed controller, we design the adaptive controller for three degree of freedom cylinder robot manipulator, which is depicted in Fig.3, where $m_{1}, m_{2}(\mathrm{~kg})$ are mass of first link and second link, $l_{1}, l_{2}(\mathrm{~m})$ are lengths of links and $\varphi(\mathrm{rad})$ is angle between first link and second link. The end effectors load $m_{2}$ is assumed to be unknown but constant, $m_{2}$ stand for parameter $\theta$. The $\tau, f_{1}, f_{2}$ are inputs as torque and force of link 1 , force of link 2 which are infected by noises $\eta_{\tau}, \eta_{f_{1}}$ and $\eta_{f_{2}}$ respectively. Physical parameters are given in Table 1.

Table 1. Physical parameters of cylinder robot manipulator

\begin{tabular}{|c|c|c|}
\hline Name & Notation & Dimension \\
\hline Mass of link 1 & $m_{1}=1$ & $(\mathrm{~kg})$ \\
\hline Mass of link 1 & $m_{1}=1.5$ & $(\mathrm{~kg})$ \\
\hline Inertial moment & $J=0.5$ & $\left(\mathrm{~m}^{2} \mathrm{Kg}\right)$ \\
\hline Acceleration of gravity & $g=9.8$ & $\left(\mathrm{~m} / \mathrm{s}^{2}\right)$ \\
\hline
\end{tabular}

The cylinder robot manipulator has output vector input vector $\underline{y}\left(\varphi l_{1} l_{2}\right)^{T}$, and input

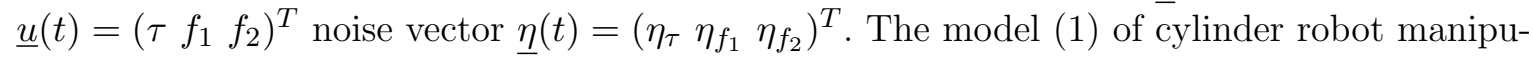
lator is given as [7]

$$
M=\left[\begin{array}{ccc}
J+\theta l_{2}^{2} & 0 & 0 \\
0 & m_{1}+\theta & 0 \\
0 & 0 & \theta
\end{array}\right] ; \underline{g}=\left[\begin{array}{c}
2 \theta l_{2} \frac{d l_{2}}{d t} \frac{d \varphi}{d t} \\
\left(m_{1}+\theta\right) g \\
-\theta l_{2} \frac{d^{2} \varphi}{d t^{2}}
\end{array}\right] .
$$

Fig. 3. Three degree cylinder robot manipulator

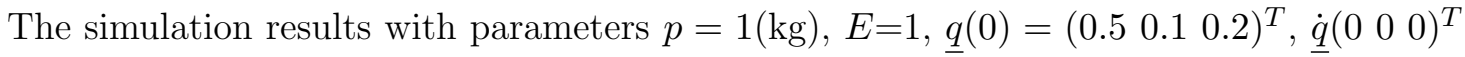
are plotted in Figures $4-10$.

In these simulations, the torques applied to joints, force applied to link 1 are affected by noises, which are described in Figures 4 and 5. The selected parameter $p$ is 1 and unnecessarily closes to the uncertain parameter of mass $m_{2}=1.5 \mathrm{Kg}$. The Figures 6 and 7 show the output signals: angle without AECM $\varphi$ and angle with AECM $a=5$ and $a=80$; length $l_{1}$ without 


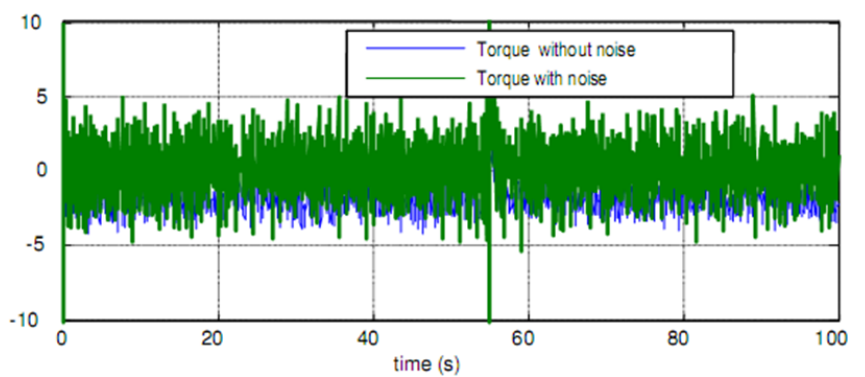

Fig. 4. Torque input of the cylinder robot manipulator

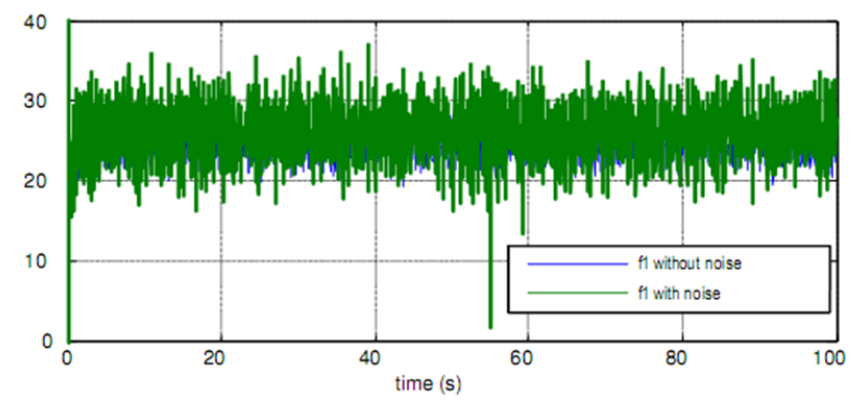

Fig. 5. Force of link 1 of the cylinder robot manipulator

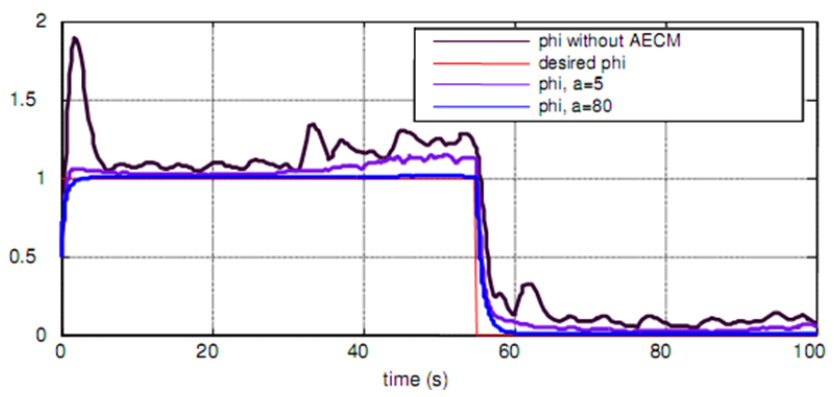

Fig. 6. Angle $\varphi$ without AECM and angle $\varphi$ with AECM

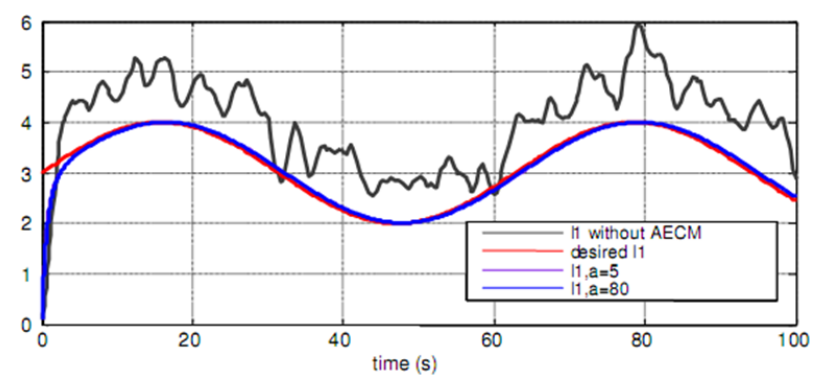

Fig. 7. Length $l_{1}$ without AECM and length $l_{1}$ with AECM $a=5$ and $a=80$

AECM and length $l_{1}$ with AECM $a=5, a=80$. The Figures 8,9 show the tracking errors and their derivatives in case $a=80$. Theoretical and simulation results indicate that the 


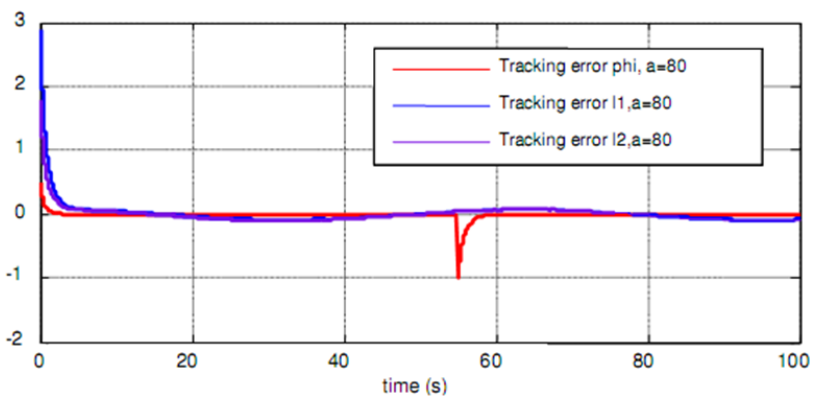

Fig. 8. Tracking errors of $\varphi, l_{1}, l_{2}$ in case $a=80$;

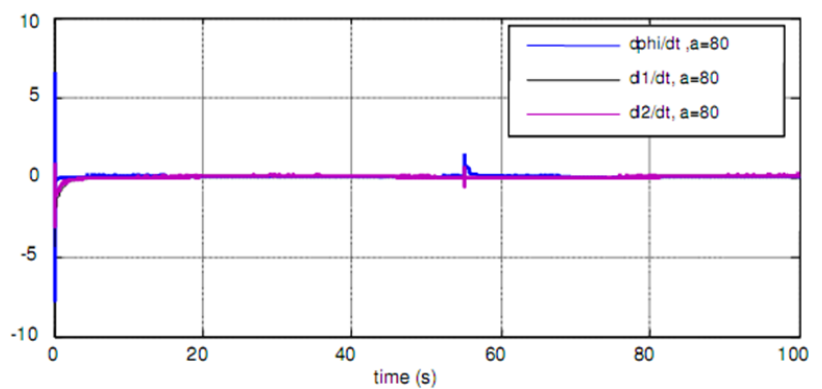

Fig. 9. Tracking errors of $d \varphi / d t, d l_{1} / d t, d l_{2} / d t$ in case $a=80$

adaptive tracking control method based on disturbance attenuation and ISS stabilization both compensates the uncertain parameter, the mass of end-effectors carried by robot manipulator, and eliminates the effect of input noises (disturbance) on robot manipulator.

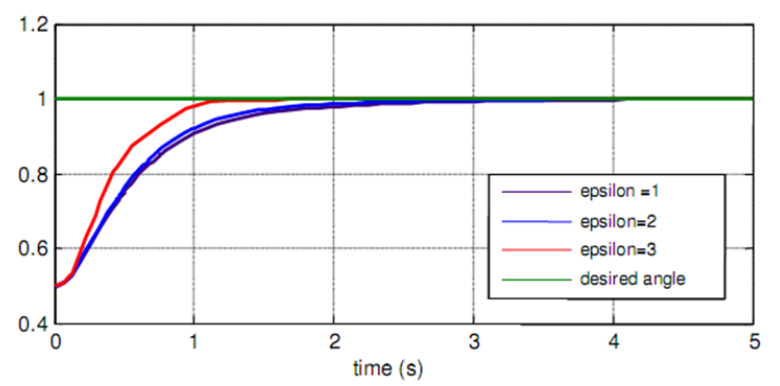

Fig. 10. Angle in case $a=30$ with $\varepsilon=1,2$ and 3

In Figure 10, we show tracking control simulation results with some transient time adjusting constant $\varepsilon=1,2$ and 3 . When we choose $\varepsilon=3$, transient time is shorter than in the cases $\varepsilon=1$ and $\varepsilon=2$.

\section{CONCLUSIONS}

This paper has proposed a new adaptive tracking control method based on disturbance attenuation and ISS stabilization of ENUI which guaranteed tracking errors. By using AECM 
and by choosing arbitrary positive parameter $p$ instead of the uncertainty parameter, positive constant $a, \varepsilon$ and matrix $E$, the tracking errors can be brought to arbitrarily small neighbourhood around the origin. This method compensates the uncertain parameters and eliminates the effect of noise on inputs of system. The advantages of this method is to converge smoothly the tracking errors to the arbitrary sufficiently small neighbourhood of the origin in optional transient time without chattering phenomenon. The simulation results have shown that the designed controller has a good behavior and that it dealt with the guaranteed tracking errors very well.

\section{REFERENCES}

[1] Nguyen Van Chi and Nguyen Doan Phuoc, Adaptive tracking control based on disturbance attenuation and ISS stabilization of Euler Lagrange nonlinear systems in the presence of uncertainty and input noise, Proceedings of the 2th IEEE Conference on Artificial Intelligent, Management Science and Electronic Commerce (AIMSEC 2011). The international workshop on Electrical Engineering and Automation Control (EEAC2011), Zhengzhou, China, Aug 8-10, 2011.

[2] Nguyen Van Chi and Nguyen Doan Phuoc, Adaptive output feedback tracking control based on Disturbance attenuation and ISS stabilization on nonlinear systems with both uncertain parameters and input noise, International symposium on Dynamic and Control ISDC2011, Hanoi, Vietnam, 19-21/sep 2011.

[3] Nguyen Van Chi, "Adaptive control for nonlinear systems by output feedback using separate principle", Doctoral thesis, Hanoi University of Science and Technology, 2011.

[4] R. Ortega, A. Loria, P. J. Nicklasson, and H. S. Ramirez, Passivity based Control of Euler Lagrange Systems, Springer-Verlag, 1998.

[5] D.M. Dawson, Robust control for the tracking of robot motion, Int. J. of Control $\mathbf{5 2}$ (3) (1990) 581-595.

[6] W. Li and J. Stoline, Parameter estimation strategies for robot applications, ASME Winter Annual Meeting, Boston, 1987.

[7] F.R. Lewits, D.M. Dawson, and C.T. Abdallah, Robot manipulator Control Theory and Practice (2. Ed) Marcel Dekker Inc., 2004.

[8] Nguyen Doan Phuoc, Advanced Control Theory, Science and Technicque Publishing House, Vietnam, 2008.

[9] S. Satry, Nonlinear System: Analysis, Stability and Control, Springer-Verlag, New York, Inc., 1999.

[10] E.D. Sontag, On the input to state stability property, European J. Control 1 (1995) 24-36.

Received on Octobor 26, 2012

Revised on May 28, 2013 